\title{
Analysis of Unwanted Stimulated Raman Scattering in Fibre Optical Parametric Oscillators
}

\author{
Han Kai* \\ College of OptoElectronic Science and Engineering, National University of Defense \\ Technology,Changsha, Hunan 410073, China \\ * email: hankai0071@126.com
}

Keywords: Stimulated Raman scattering; Optical parametric oscillators; Four wave mixing;

\begin{abstract}
The Stimulated Raman Scatting (SRS) sometimes plays a negative role in a fibre OPO ring cavity, which may consume the signal laser power. We established a mathematical model for describing the SRS process in the OPO cavity. A SRS threshold is presented. The SRS net effective gain should be smaller than 16.5 in general. The corresponding experiment shows accordant results. The SRS suppression method is discussed at the end. Employing longer and larger delay fibre is an effective and convenient measure, while the currently available commercial WDM may not be competent for the SRS suppression in the OPO cavity.
\end{abstract}

\section{Introduction}

Nonlinear fiber lasers and amplifiers such as optical parametric oscillators (OPOs) and Raman amplifiers offer wavelength agility and a number of other attractions relative to their more widespread rare-earth doped counterparts [1-3]. Disadvantages include generally much higher pump power requirements, but also unwanted nonlinear scattering, which can make it difficult to spectrally confine radiation. This is difficult to address through basic fiber parameters such as length and core area, since the nonlinear scattering is typically of the third order and thus scale similarly with those fiber parameters. The problem is particularly bad when the threshold for the unwanted process is much lower than that of the desired process, as in the case of Raman amplification of narrow line signals. [2] For these, undesired stimulated Brillouin scattering occurs much more readily than the desired stimulated Raman scattering (SRS). Similarly, because the effective area of the signal is much smaller than the effective area of the pump in a claddingpumped fiber Raman laser, often-undesired 2nd-order SRS can occur more readily than the desired 1st-order SRS.

In this paper, we theoretically investigate the use of a fibre delay line and spectral filtering to suppress unwanted SRS from the anti-Stokes signal in a synchronously-pumped pulsed OPO. OPOs are attractive for anti-Stokes generation because of the relatively low threshold that an oscillator configuration can potentially offer. A synchronously pumped configuration, a delay line can be used to reduce the average power required even further by reducing the cavity round-trip frequency. However, although the four-wave mixing (FWM) that the OPOs rely upon can generate higher gain than the competing SRS, it is difficult to maintain phase-matching over the long nonlinear fibre converters required for low-threshold OPOs. This reduces the parametric gain and makes the unwanted SRS more difficult to control, including SRS generated in the delay line. However, for sufficiently large fibre cross sectional area, we find that the net effect of the delay line is to suppress the SRS rather than to add to it. The currently available commercial WDM has some ability for the SRS suppression but it is not adequate due to its limited wavelength distinguishing ability. Although we have focused on a specific appeal configuration, the results are applicable to a range of other nonlinear devices such as cladding-pumped Raman lasers. 


\section{Mathematical model}

Although SRS is a meaningful nonlinear process in Raman amplifiers/lasers, it may become unwelcome in a fibre OPO ring cavity which limits the signal laser power. In general, the fibre OPO ring cavity is composed of nonlinear fibre and delay fibre. The signal laser intensity distribution, SRS gain and fibre loss is normally different in different segments of the cavity. The experiment setup in [4] shown in Fig.1 is taken as an example to analyse the unwanted SRS suppression. The photonic crystal fibre (PCF-LMA8, NKT Photonics) is employed as the nonlinear medium, in which the pump laser (1079nm narrow linewidth pules fibre laser, self-made) converts to signal laser based on four-wave mixing (FWM). A delay line (Hi1060, Corning) ensures that the roundtrip time of the OPO cavity can be synchronized to the pump's pulse repetition frequency. The pigtails of coupler and WDM are the same as the delay fibre. Additionally, the laser polarization has been mostly preserved in the OPO cavity. In the experiment, obvious SRS is observed, which is pumped by the FWM anti-Stokes as the waves propagate through the delay line fibre.

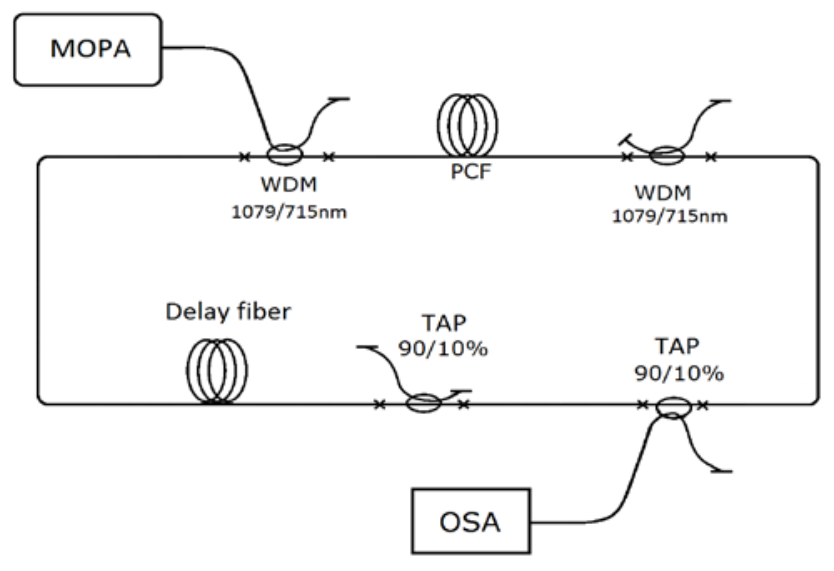

Fig.1 OPO cavity configuration

For convenience, the OPO cavity is divided into two segments: PCF and delay fibre, which have different effective cross sectional area Aeff, SRS gain coefficient $g_{R}$ and attenuation constant $\alpha$. One-dimensional spatial coordinates is established along the OPO cavity, while the origin of coordinates is set at the beginning of the PCF. The effective cross sectional area $A_{\text {eff }}(\mathrm{z})$ and the SRS gain coefficient $g_{R}(\mathrm{z})$ can be expressed as,

$$
\begin{gathered}
A_{\text {eff }}(z)=\left\{\begin{array}{lc}
A_{P C F} & 0 \leq z<L_{P C F} \\
A_{\text {delay }} & L_{P C F} \leq z<L_{0}
\end{array}\right. \\
g_{R}(z)=\left\{\begin{array}{lr}
g_{R, P C F} & 0 \leq z<L_{P C F} \\
g_{R, \text { delay }} & L_{P C F} \leq z<L_{0}
\end{array}\right.
\end{gathered}
$$

The effective cross sectional area Aeff, the SRS gain coefficient $g_{R}$ and the fibre length $L$ are labelled with subscript 'PCF', which refer to PCF, while they are labelled with subscript 'delay', which refer to delay fibre. Here, $L_{0}=L_{P C F}+L_{\text {delay }}$, which denotes the total length of the OPO cavity.

The discrepancy on fibre loss between the SRS Stokes wave and the SRS pump wave is ignored. The attenuation constant $\alpha(z)$ of the cavity can be written as,

$$
\alpha(z)=\left\{\begin{array}{lr}
\alpha_{1} \cdot \delta(z) & z=0 \\
\alpha_{P C F} & 0<z<L_{P C F} \\
\alpha_{2} \cdot \delta\left(z-L_{P C F}\right) & z=L_{P C F} \\
\alpha_{\text {delay }} & L_{P C F}<z<L_{0}
\end{array}\right.
$$

Here, $\alpha_{1}$ and $\alpha_{2}$ represent the non-neglectable splice loss between the two types of fibre.

The FWM anti-Stokes acts as pump wave in the establishment of SRS, so it is labelled with $P_{P}$, which is supposed to exponentially increase in the nonlinear fibre [4-6]. It is given by, 


$$
P_{p}(z)=\left\{\begin{array}{lr}
p_{0} \cdot \frac{\cosh \left(g_{F W M} \cdot z\right)}{\cosh \left(g_{F W M} \cdot L_{P C F}\right)} & 0 \leq z<L_{P C F} \\
p_{0} \cdot e^{-\alpha_{2}-\alpha_{\text {delay }} \cdot\left(z-L_{P C F}\right)} & L_{P C F} \leq z<L_{0}
\end{array}\right.
$$

Here, $p_{0}$ is the FWM anti-Stokes peak power at the end of the PCF; the $g_{F W M}$ is the net gain of the anti-Stokes light in PCF. Considering the periodicity of the ring cavity, there are,

$$
\begin{gathered}
A_{\text {eff }}(z)=A_{\text {eff }}\left(z+L_{0}\right) \\
g_{R}(z)=g_{R}\left(z+L_{0}\right) \\
\alpha(z)=\alpha\left(z+L_{0}\right) \\
P_{P}(z)=P_{P}\left(z+L_{0}\right)
\end{gathered}
$$

The average value of the pump peak power, cavity loss, peak gain coefficient and the effective mode area are given by,

$$
\begin{gathered}
\overline{p_{p_{1}}}=\frac{1}{L_{P C F}} \cdot \int_{0}^{L_{P C F}} p_{0} \frac{\cosh \left(g_{F W M} \cdot z\right)}{\cosh \left(g_{F W M} \cdot L_{P C F}\right)} d z=p_{0} \cdot \frac{\tanh \left(g_{F W M} \cdot L_{P C F}\right)}{g_{F W M} \cdot L_{P C F}} \\
\overline{p_{p_{2}}}=\frac{1}{L_{\text {delay }}} \cdot \int_{L_{P C F}}^{L_{0}} p_{0} \cdot e^{-\alpha_{2}-\alpha_{\text {delay }} \cdot\left(z-L_{P C F}\right)} d z=p_{0} \cdot e^{-\alpha_{2}} \cdot \frac{1-e^{-\alpha_{\text {delay }} \cdot L_{\text {delay }}}}{\alpha_{\text {delay }} \cdot L_{\text {delay }}} \\
\overline{p_{p}}=\frac{1}{L_{0}} \cdot \int_{0}^{L_{0}} p_{p}(z) d z=t_{1} \cdot \overline{p_{p_{1}}}+t_{2} \cdot \overline{p_{p}} \\
\bar{\alpha}=\frac{1}{L_{0}} \cdot \int_{0}^{L_{0}} \alpha(z) d z \\
\overline{g_{R}}=\frac{1}{L_{0}} \cdot \int_{0}^{L_{0}} g_{R}(z) d z=t_{1} \cdot g_{R, P C F}+t_{2} \cdot g_{R, \text { delay }} \\
\overline{A_{\text {eff }}}=\frac{1}{L_{0}} \cdot \int_{0}^{L_{0}} A_{e f f}(z) d z=t_{1} \cdot A_{P C F}+t_{2} \cdot A_{\text {delay }}
\end{gathered}
$$

Parameter $t_{1}$ and $t_{2}$ are defined to characterize the proportion of the PCF and the delay fibre in the cavity, respectively.

$$
\begin{gathered}
t_{1}=\frac{L_{P C F}}{L_{0}} \\
t_{2}=\frac{L_{\text {delay }}}{L_{0}}
\end{gathered}
$$

Due to the group-velocity dispersion effect, the Raman Stokes pulse generally walks-off from pump pulse in the non-zero dispersion regime. The walk-off length $L_{W}$ is expressed as, [6]

$$
L_{W}=\frac{T}{\beta_{1 p}-\beta_{1 s}} \approx-\frac{T}{D\left(\lambda_{p}\right) \cdot \Delta \lambda}=-\frac{T}{\left[t_{1} \cdot D_{P C F}\left(\lambda_{p}\right)+t_{2} \cdot D_{\text {delay }}\left(\lambda_{p}\right)\right] \cdot \Delta \lambda}
$$

Where, $\mathrm{T}$ is the SRS pump pulse full width at half maximum (FWHM); $\beta_{1 p}$ and $\beta_{1 s}$ are the group velocity reciprocal of the SRS pump wave and SRS Stokes wave; $\Delta \lambda=\lambda_{s}-\lambda_{p}$ is the wavelength interval of the SRS Stokes wave and SRS pump wave; $D_{P C F}\left(\lambda_{p}\right)$ and $D_{\text {delay }}\left(\lambda_{p}\right)$ are the dispersion parameter of the nonlinear fibre and the delay fibre at the pump wavelength, respectively.

The pump pulse is assumed to be Gaussian profile [7]. The net effective SRS gain in the cavity $G_{n e t}$ is expressed as,

$$
G_{\text {net }}=\int_{-L_{\text {eff }} / 2+Z^{\prime}}^{L_{e f f} / Z^{\prime}} \frac{g_{R}(z) \cdot P_{P}(z)}{A_{\text {eff }}(z)} \cdot \exp \left[-\left(\frac{T}{\tau}\right)^{2} \cdot\left(\frac{z-z^{\prime}}{L_{W}}\right)^{2}\right]-\alpha(z) d z
$$

Where, $\tau$ is the $1 / e$ Gaussian half width of the pulse, which is 1.665 times smaller than the FWHM i.e. $T=1.665 \tau$. $z$ ' represents the position at which the potential Stokes meets the peak of 
the pump; $L_{\text {eff }}$ is defined as the effective interaction length, in which, the SRS gain is larger than the loss of the SRS Stokes all along.

$$
L_{\text {eff }}=2 \cdot \frac{\tau}{T} \cdot L_{W} \cdot \sqrt{\ln \left(\frac{\overline{g_{R}} \cdot \overline{p_{P}}}{\bar{\alpha} \cdot \overline{A_{\text {eff }}}}\right)}
$$

According to equation (19), in the case of small pump power or large cavity loss, which means the SRS Stokes gain becomes smaller than loss before the Stokes pulse walk-off from the pump pulse, the effective interaction length is smaller than the walk-off length. In case of tiny cavity loss or large pump power, which means the SRS Stokes gain is still larger than the loss even when the separation between the Stokes pulse and the pump pulse exceeds the walk-off length, the effective interaction length is larger than the walk-off length. If $\left(\overline{g_{R}} \cdot \overline{p_{P}}\right) /\left(\bar{\alpha} \cdot \overline{A_{e f f}}\right)<1$, which means the SRS gain is smaller than the loss all the time and $G_{n e t}<0$, the SRS cannot occur.

It is assumed that the SRS pump remains undepleted in the SRS process. The Raman threshold condition is defined as that the SRS Stokes power reaches one percent of the SRS pump power i.e. the SRS Stokes power is 20db smaller than the SRS pump power. According to the reference [7-8], the SRS threshold condition is written as.

$$
\begin{gathered}
p_{s}(\text { eff }) \cdot e^{G_{\text {net }}}=\overline{p_{p}} / 100 \\
p_{s}(e f f)=h v_{s} \cdot B_{e f f} \\
B_{\text {eff }}=\frac{\sqrt{\pi}}{2} \cdot \delta v_{\mathrm{FWHM}} \cdot G_{\text {net }}^{-1 / 2}
\end{gathered}
$$

Where, $p_{s}($ eff $)$ is the initial SRS Stokes power, which builds up from spontaneous Raman scattering occurring throughout the cavity; $B_{\text {eff }}$ is the effective bandwidth or the longitudinal mode number of the Stokes radiation; [8] $h$ is Planck constant; $v_{s}$ is the frequency of the SRS Stokes wave; $\delta v_{\mathrm{FWHM}}$ is the full width at half maximum of the spontaneous Raman gain spectrum.

In our OPO ring cavity, the nonlinear fibre (PCF-LMA8, NKT Photonics) is of 35m length with effective cross sectional area $38.48 \mu \mathrm{m}^{2}$, SRS gain coefficient $2 \times 10^{-13} \mathrm{~m} / \mathrm{w}$ and fibre loss $4 \mathrm{~dB}$ (bended PCF), i.e. $L_{P C F}=35 \mathrm{~m}, A_{P C F}=38.48 \mu \mathrm{m}^{2}, g_{R, P C F}=2 \times 10^{-13} \mathrm{~m} / \mathrm{w}$ and $\alpha_{P C F}=2.63 \times 10^{-2} \mathrm{~m}^{-1}$; the delay fibre (Hi1060, Corning) is of $65 \mathrm{~m}$ length with effective cross sectional area $19.05 \mu \mathrm{m}^{2}$, SRS gain coefficient $6 \times 10^{-14} \mathrm{~m} / \mathrm{w}$ and fibre loss $0.26 \mathrm{~dB}$, i.e. $L_{\text {delay }}=65 \mathrm{~m}, A_{\text {delay }}=19.05 \mu \mathrm{m}^{2}$, $g_{R \text {, delay }}=6 \times 10^{-14} \mathrm{~m} / \mathrm{w}$ and $\alpha_{\text {delay }}=9.21 \times 10^{-4} \mathrm{~m}^{-1}$. The splice loss between PCF and delay fibre at $z=0$ and $z=L_{P C F}$ are $3.5 \mathrm{~dB}$ and $4.5 \mathrm{~dB}$, respectively. According to literature[4], the anti-Stokes, which acts as SRS pump here, is of peak power $p_{0} 112 w$ with pulse width (FWHM) $T$ 840ps and the net gain of the anti-Stokes $g_{F W M}$ is taken as $0.074 m^{-1}$. The wavelength of the SRS pump wave and the SRS Stokes wave are $715 \mathrm{~nm}$ and $738 \mathrm{~nm}$, then the wavelength interval is $23 \mathrm{~nm}$. The dispersion parameter of the nonlinear fibre and the delay fibre are $-150 \mathrm{ps} / \mathrm{km} / \mathrm{nm}$ and $-110 \mathrm{ps} / \mathrm{km} / \mathrm{nm}$ at $715 \mathrm{~nm}$. Based on equation (1)-(19), the $\overline{p_{p}}, \bar{\alpha}, \overline{g_{R}}, \overline{A_{\text {eff }}}, L_{W}$, and $L_{\text {eff }}$ are calculated to be $40.05 \mathrm{w}, 2.82 \times 10^{-2} \mathrm{~m}^{-1}, 1.09 \times 10^{-13} \mathrm{~m} / \mathrm{w}, 25.85 \mathrm{\mu m}^{2}, 295 \mathrm{~m}$, and $476 \mathrm{~m}$ respectively. Since the effective interaction length $L_{\text {eff }}$ covers several cavity round trips, the SRS Stokes and the net effective SRS gain $G_{n e t}$ can be simplified.

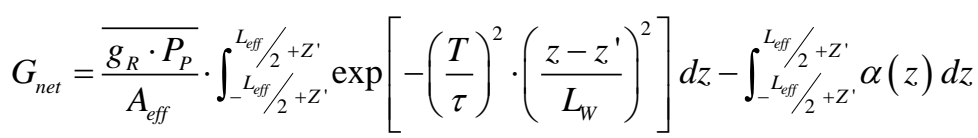

Here, $\overline{g_{R} \cdot P_{P} / A_{\text {eff }}}$ is the average value of $g_{R}(z) \cdot P_{P}(z) / A_{\text {eff }}(z)$ in one round trip.

$$
\frac{\overline{g_{R} \cdot P_{P}}}{A_{\text {eff }}}=\frac{1}{L_{0}} \cdot \int_{0}^{L_{0}} \frac{g_{R}(z) \cdot P_{P}(z)}{A_{e f f}(z)} d z=t_{1} \cdot \frac{g_{R, P C F} \cdot \overline{p_{p_{1}}}}{A_{P C F}}+t_{2} \cdot \frac{g_{R, \text { delay }} \cdot \overline{p_{p_{2}}}}{A_{\text {delay }}}
$$


As a general assumption, the potential Stokes meets the pump pulse peak at the position of $\mathrm{z}=0$, that means $z^{\prime}=0$ and $G_{n e t}$ can be further simplified to be,

$$
G_{n e t} \approx \sqrt{\pi} \cdot \frac{\tau}{T} \cdot \operatorname{erf}\left(\frac{1}{2} \cdot \frac{T}{\tau} \cdot \frac{L_{\text {eff }}}{L_{w}}\right) \cdot\left(t_{1} \cdot \frac{\overline{p_{p_{1}}} \cdot g_{R, P C F}}{A_{P C F}}+t_{2} \cdot \frac{\overline{p_{p_{2}}} \cdot g_{R, \text { delay }}}{A_{\text {delay }}}\right) \cdot L_{w}-\bar{\alpha} \cdot L_{\text {eff }}
$$

Considering the relationship between $T$ and $\tau$,

$$
G_{\text {net }}=1.06 \cdot \operatorname{erf}\left(0.83 \cdot \frac{L_{e f f}}{L_{w}}\right) \cdot\left(t_{1} \cdot \frac{\overline{p_{p_{1}}} \cdot g_{R, P C F}}{A_{P C F}}+t_{2} \cdot \frac{\overline{p_{p_{2}}} \cdot g_{R, \text { delay }}}{A_{\text {delay }}}\right) \cdot L_{w}-\bar{\alpha} \cdot L_{\text {eff }}
$$

The width of spontaneous Raman gain spectrum $\delta v_{\text {FWHM }}$ is taken as $4.64 \times 10^{11} \mathrm{~Hz}$. In the undepleted-pump approximation, according to the SRS threshold condition (20)-(23) the net effective SRS gain $G_{\text {net }}$ should be smaller than 16.5 to mitigate the SRS in OPO ring cavity, that is

$$
1.06 \cdot \operatorname{erf}\left(0.83 \cdot \frac{L_{e f f}}{L_{w}}\right) \cdot\left(t_{1} \cdot \frac{\overline{p_{p_{1}}} \cdot g_{R, P C F}}{A_{P C F}}+t_{2} \cdot \frac{\overline{p_{p_{2}}} \cdot g_{R, \text { delay }}}{A_{\text {delay }}}\right) \cdot L_{w}-\bar{\alpha} \cdot L_{\text {eff }}<16.5
$$

For the range of parameters involved here, the SRS threshold condition is given to a very good approximation by equation (27).

In literature [4], the signal laser peak power $\mathrm{p} 0$ reaches $112 \mathrm{w}$. According to equation (26), the SRS net effective gain achieves 32, which is much larger than the threshold 16.5. Correspondingly, serious cascaded SRS has arisen, as shown in figure 7 in [4]. To demonstrate the SRS process under various SRS pump power, the signal laser peak power p0 is reduced to $88.2 \mathrm{w}, 77.8 \mathrm{w}, 67.4 \mathrm{w}, 57.1 \mathrm{w}$ and 36.3w by lowering the FWM pump power. Based on equation (9)-(14) and (26), the SRS net gain become 24.2, 19.2, 14.9, 11.1 and 3.8, respectively, and the corresponding spectra is recorded, which is shown in Fig. 2

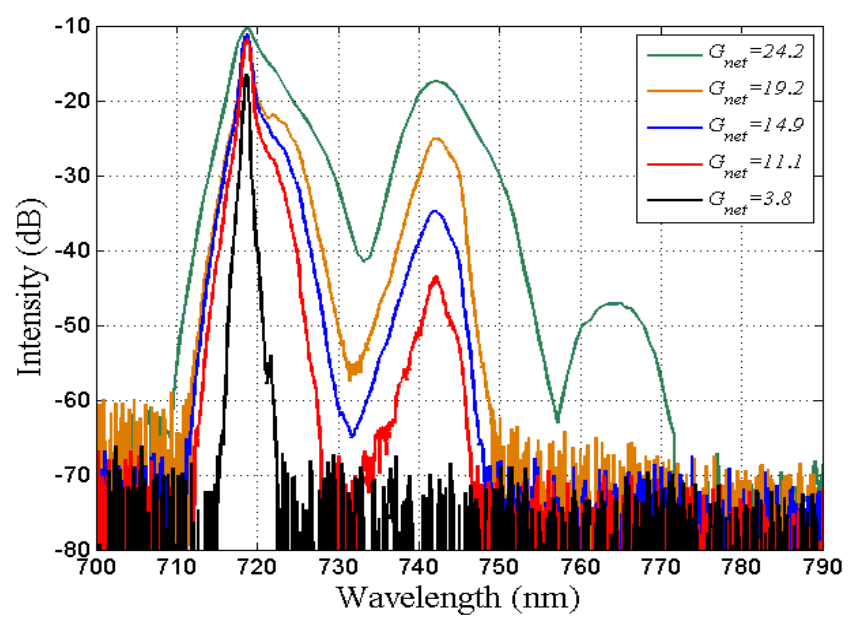

Fig. 2 the SRS spectra at various SRS net gain in the OPO cavity

As illustrated in Fig. 2, the SRS Stokes power is $7.3 \mathrm{~dB}, 13.6 \mathrm{~dB}, 23.5 \mathrm{~dB}, 31.7 \mathrm{~dB}$, and $70+\mathrm{dB}$ smaller than the SRS pump power. In other words, in the case of SRS net gain larger than 16.5, intense SRS Stokes appear in the OPO cavity, while in the case of SRS net gain smaller than 16.5 the SRS Stokes power is neglectable compared with the SRS pump power Compared with the SRS pump power, the SRS Stokes is smaller by more than two order magnitude (20dB).. The validity of equation is confirmed by Fig.2, to some extent.

\section{Discussion of SRS suppression in OPO cavity}

It is obviously unwise to suppress the unwelcome nonlinear effect at the cost of the desired signal power. It is quite necessary to explore a practical method to mitigate the unwanted nonlinear effect in the OPO cavity. The delay fibre is the most flexible part in the OPO cavity. Thus we studied the SRS net effective gain in the OPO cavity with various kinds of delay fibre involved. 
According to equation (1-18), the dependence of the SRS net gain on the delay fibre length is figured out and the result is shown in Fig.3.

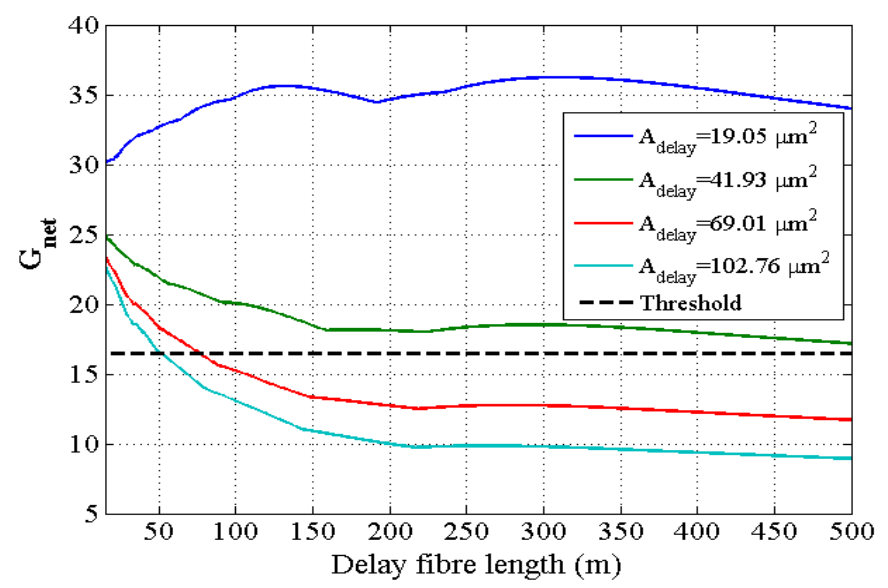

Fig. 3 Dependence of the SRS net effective gain on the delay fibre length

It should be noticed that the equation (18) is an integration of a piecewise non-continuous function, which can be written as a summation of a series of continuous integrations. The effective interaction length $L_{\text {eff }}$ extends with the delay fibre length increase. That means the integral boundary increases and increasing integral pieces are involved. Every inflection point means a new integral piece is involved. In other words, each inflection point in the unsmooth curves in Fig. 3 declares a new fibre segment starts contributing to the SRS.

In the case that the delay fibre effective cross sectional area are $19.05 \mu \mathrm{m} 2,41.93 \mu \mathrm{m} 2,69.01 \mu \mathrm{m} 2$, $102.76 \mu \mathrm{m} 2$ individually, the SRS net effective gain in the OPO cavity is demonstrated in Fig3. For the $69.01 \mu \mathrm{m} 2$ and $102.76 \mu \mathrm{m} 2$ delay fibre, the SRS net effective gains fall below the threshold 16.5 when their length is larger than $78 \mathrm{~m}$ and $51 \mathrm{~m}$. As illustrated in Fig.3, it is practicable to suppress the unwelcome SRS in the OPO cavity by using longer and thicker delay fibre.

Another possible method for the SRS suppression is to employ wavelength-division multiplexer (WMD) to separate the SRS pump laser and the SRS Stokes laser. Considering the tiny wavelength interval, the available commercial WDM has limited distinguishing ability for the SRS pump laser and the SRS Stokes laser. In our case, we take a kind of special WDM(Gooch\&Housego) as an example, the insertion losses for the SRS pump and the SRS Stokes are $0.17 \mathrm{~dB}$ and $0.34 \mathrm{~dB}$ respectively. Taking the WDM insertion loss into account, the SRS net gain is figured out and shown in Fig.4.

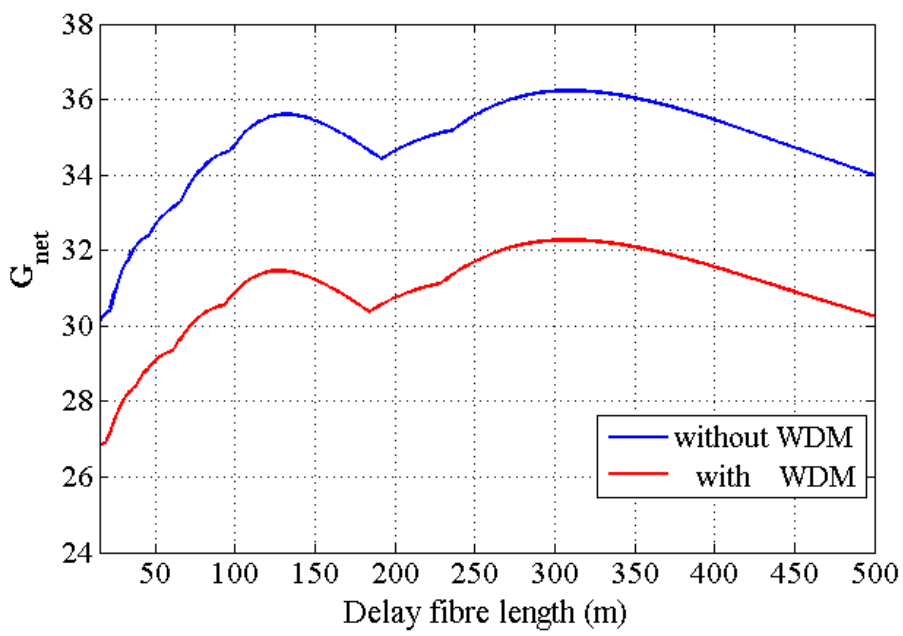

Fig. 4. Dependence of the SRS net effective gain on the delay fibre length with WDM

As illustrated in Fig. 4, the WDM decreased the SRS net effective gain in the OPO cavity. However the reduction is so limited that the SRS net gain is still above the threshold. The currently available commercial WDM is not competent to suppress the SRS alone. 


\section{Conclusion}

We established a mathematical model to analyse the unwanted SRS in the OPO cavity, in which the complex cavity configuration, SRS contributions of different fibre segments and the pulse dispersion are taken into account. The SRS threshold in an OPO cavity is presented by our mathematical model. As long as the SRS net effective gain is below the threshold, the unwanted SRS remains negligible in the OPO cavity. Accordant experiment results are obtained which confirm the validity of our mathematical modal. Considering the delay fibre is the most flexible part in the OPO cavity, longer and larger delay fibre is an effective and convenient measure for the SRS suppression in the OPO cavity. The currently available commercial WDM is able to suppress the SRS to some extent. Nevertheless, its performance may not be adequate due to its limited distinguishing ability. The analysis for the suppression of the unwanted nonlinear effects is of great help to optimize the OPO cavity configuration and achieve higher signal power.

\section{Reference}

[1] J. Nilsson, J. K. Sahu, J. N. Jang, R. Selvas, D. C. Hanna, and A. B. Grudinin, "Claddingpumped Raman amplifier,” in Proc. OAA (2002), Vancouver, Canada, Jul. 14-17, 2002, Paper PD2.

[2] J. Ji, C. A. Codemard, J. K. Sahu, M. Ibsen, and J. Nilsson, “Analysis of the conversion to the 1st Stokes in cladding-pumped fiber Raman amplifiers,” IEEE J. Sel. Top. Quantum Electron, vol. 15, no. 1, pp. 129-139.(2009)

[3] Junhua Ji, Christophe A. Codemard, and Johan Nilsson, ”Analysis of Spectral Bendloss Filtering in a Cladding-Pumped W-Type Fiber Raman Amplifier," J Lightwave Technology, VOL. 28, NO. 15, pp. 2179-2186. (2010)

[4] Gys Van der Westhuizen and Johan Nilsson, "Fiber Optical Parametric Oscillator for Large Frequency-Shift Wavelength Conversion” IEEE J. of Quantum Electron. VOL. 47, NO. 11, pp. 1396-1403.

[5] R.H. Stolen and J.E. Bjorkholm, "Parametric amplification and frequency conversion in optical fibers,” IEEE J. of Quantum Electron. QE-18, 1062-1072 (1982).

[6] G. P. Agrawal, Nonlinear fiber optics (Fourth Edition) (Academic Press, 2007)

[7] R.H. Stolen, and A. M. Johnson, The Effect of Pulse Walkoff on Stimulated Raman Scattering in FibersIEEE JOURNAL OF QUANTUM ELECTRONICS 1986

[8] Smith, R G, Optical Power Handling Capacity of Low Loss Optical Fibers as Determined by Stimulated Raman and BrillouinScatteringApplied Optics 1972 